\title{
ERS International Congress, Madrid, 2019: highlights from the Pulmonary Vascular Diseases Assembly
}

\author{
Sheila Ramjug ${ }^{1}$, Jason Weatherald $\mathbb{1}^{2,3}$, Sandeep Sahay $\mathbb{1}^{4}$, Johad Khoury $\mathbb{1}^{5}$, \\ Vasile Foris ${ }^{6,7}$, Nagaraj Chandran ${ }^{6,7}$, Aleksandar Bokan (10 ${ }^{8}$, Laurent Godinas ${ }^{9}$ \\ and Marion Delcroix (10)
}

\begin{abstract}
Affiliations: ${ }^{1}$ Dept of Respiratory Medicine, Manchester University NHS Foundation Trust, Wythenshawe, UK. ${ }^{2}$ Dept of Medicine, Division of Respirology, University of Calgary, Calgary, AB, Canada. ${ }^{3}$ Libin Cardiovascular Institute of Alberta, University of Calgary, Calgary, AB, Canada. ${ }^{4}$ Houston Methodist Lung Center, Division of Pulmonary Medicine, Houston Methodist Hospital, Houston, TX, USA. ${ }^{5}$ Pulmonary Division, Lady Davis-Carmel Medical Center, Haifa, Israel. 'Medical University of Graz, Dept of Internal Medicine, Division of Pulmonology, Graz, Austria. ${ }^{7}$ Ludwig Boltzmann Institute for Lung Vascular Research, Graz, Austria. ${ }^{8}$ Dept for Emergency Pulmonology, Institute for Pulmonary Diseases of Vojvodina, Faculty of Medicine Novi Sad, Novi Sad, Serbia. ${ }^{9}$ Dept of Respiratory Diseases, UZ Leuven, Leuven, Belgium.
\end{abstract}

Correspondence: Sheila Ramjug, Manchester University NHS Foundation Trust, Southmoor Road, Wythenshawe, M23 9LT, UK. E-mail: sheilaramjugahotmail.com

ABSTRACT The 2019 European Respiratory Society (ERS) International Congress, held in Madrid, Spain, had exciting sessions regarding the field of pulmonary vascular disease. The symposia related to the new ERS/European Society of Cardiology (ESC) Guidelines for the diagnosis and management of acute pulmonary embolism were well received, as were sessions on pulmonary hypertension related to lung disease, demonstrating the concept of pulmonary hypertension not being the rarity that it was previously thought to be. The use of risk stratification in relation to pulmonary arterial hypertension (PAH) was heavily featured and the scientific sessions informing the respiratory community of potential biomarkers and targets for future therapies were thought-provoking.

This article discusses highlights of the 2019 pulmonary vascular disease sessions as a summary of current knowledge and practice. We have summarised the key points from the sessions pertaining to the new ERS/ESC Guidelines for the management of acute pulmonary embolism. We have also focused on prognostic factors and potential therapies in pulmonary hypertension related to interstitial lung disease. Relating to $\mathrm{PAH}$, we have reviewed the symposia on risk stratification, along with the use of noninvasive measures and the sessions relating to biomarkers in PAH.

@ERSpublications

This article aims to summarise research presented at \#ERSCongress 2019: the new @escardio/ @EuroRespSoc guidance on acute PE diagnosis and management, $\mathrm{PH}$ in relation to chronic lung disease, and advances in pulmonary arterial hypertension https://bit.ly/3bAUG0o

Cite this article as: Ramjug S, Weatherald J, Sahay S, et al. ERS International Congress, Madrid, 2019: highlights from the Pulmonary Vascular Diseases Assembly. ERJ Open Res 2020; 6: 00304-2020 [https://doi.org/10.1183/23120541.00304-2020].

Received: 25 May 2020 | Accepted after revision: 28 Aug 2020

Copyright $\odot$ ERS 2020. This article is open access and distributed under the terms of the Creative Commons Attribution Non-Commercial Licence 4.0. 


\section{Acute pulmonary embolism}

The new ERS/ESC Guidelines for the diagnosis and management of acute pulmonary embolism

During the 2019 European Respiratory Society (ERS) International Congress in Madrid, Spain, there was a huge focus on pulmonary embolism (PE) sessions, which was well received by Congress delegates as it coincided with the recent publication and update of the ERS/European Society of Cardiology (ESC) Guidelines for the diagnosis and management of acute PE. In this and the following sections, we will focus on the updated areas in the 2019 guidance for the acute management of PE.

Stavros Konstantinides started the proceedings by reiterating the usefulness of transthoracic echocardiography (TTE) to assess the right ventricle for signs of dysfunction when faced with the haemodynamically unstable patient. In the new guidance, the definition of haemodynamic instability has also altered to include "obstructive shock", implying there is evidence of end-organ hypoperfusion with a systolic blood pressure $<90 \mathrm{mmHg}[1]$.

Fortunately, however, many patients we assess are not unstable and Prof. Konstantinides emphasised the importance of performing a Wells or Geneva score and only a D-dimer test if there is a low or intermediate probability of a venous thromboembolism (VTE) event (figure 1).

Relating to the use of D-dimer, classically we would consider a level $>500 \mathrm{ng} \cdot \mathrm{mL}^{-1}$ as raised. Since the results of the ADJUST-PE study in 2014, its use with an age-adjusted cut-off is advocated in those who are aged $\geqslant 50$ years, e.g. in a 60-year-old using a D-dimer cut-off level of $600 \mathrm{ng} \cdot \mathrm{mL}^{-1}$ [2].

The adapted D-dimer cut-off based on clinical probability, as per the YEARS study group publication in 2017 [3], has also been recommended in the current guidance. This simplified diagnostic tool utilises the YEARS clinical decision rule of 1) signs of deep venous thrombosis, 2) haemoptysis, and 3) PE more likely than an alternative diagnosis, with an adapted D-dimer cut-off dependent on the answers to the "rule" [3].

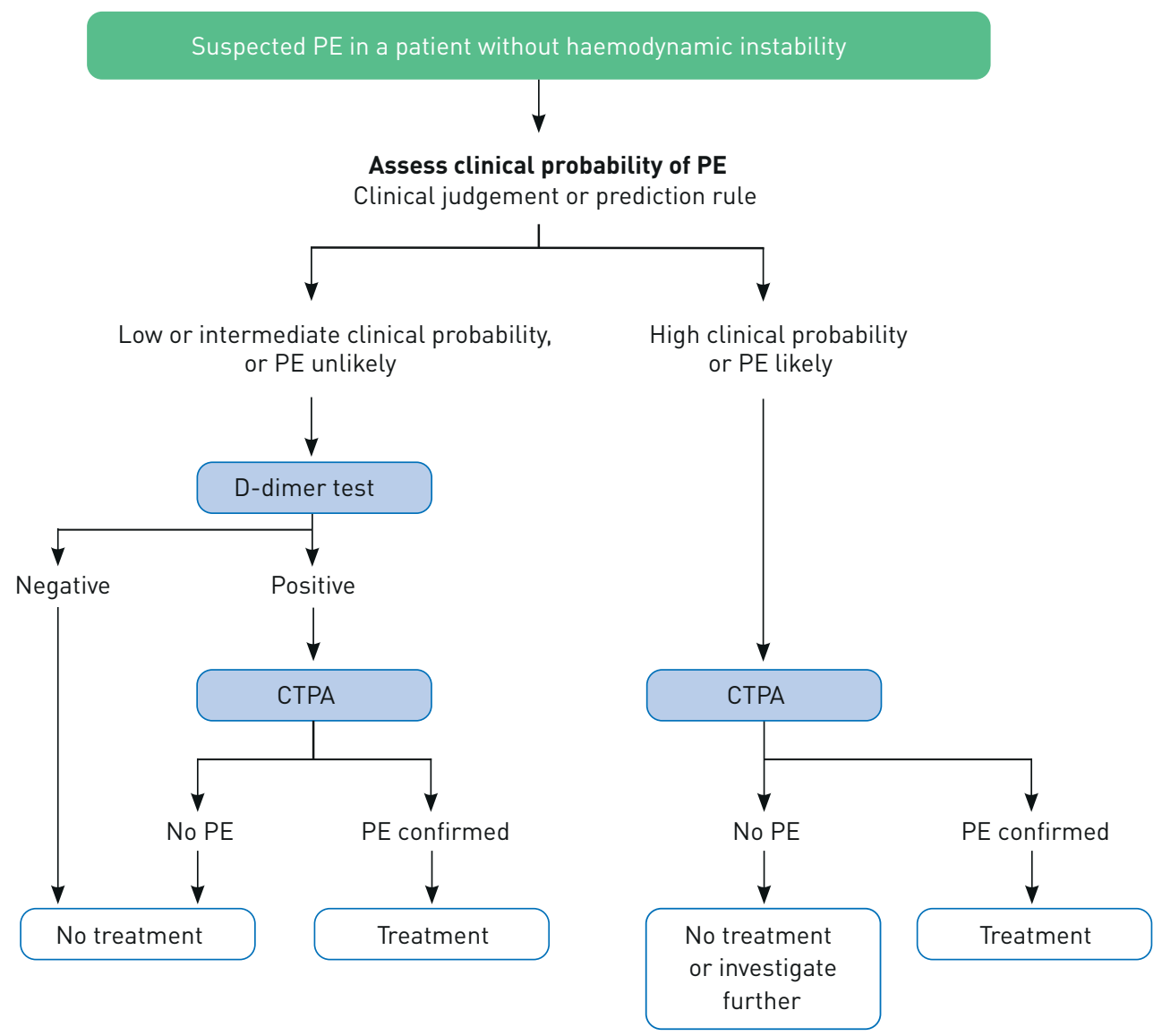

FIGURE 1 Diagnostic algorithm for patients with suspected pulmonary embolism (PE) without haemodynamic instability. CTPA: computed tomography pulmonary angiography. Reproduced and modified from [1] with permission. 
For example, if there is no evidence of these points, a D-dimer cut-off of $<1000 \mathrm{ng} \cdot \mathrm{mL}^{-1}$ is used to exclude a PE; if, however, there is a positive clinical point then a D-dimer cut-off level of $<500 \mathrm{ng} \cdot \mathrm{mL}^{-1}$ is used.

By utilising these methods, we are ensuring the judicious use of computed tomography pulmonary angiography (CTPA) in our clinical environments. It was also highlighted that we must not overlook the role of ventilation/perfusion $\left(V^{\prime} / Q^{\prime}\right)$ single-photon emission computed tomography in the diagnosis of PE. The new guidance also usefully illustrates the radiation of each imaging modality; this is especially relevant when considering female breast tissue [1].

Prof. Konstantinides discussed the importance of examining for right ventricular (RV) dysfunction, even in those with a simplified Pulmonary Embolism Severity Index (sPESI) score of 0. This stems from a meta-analysis by BARCO et al. [4] in 3000 patients with a low Pulmonary Embolism Severity Index (PESI), or sPESI of 0 . The investigators discovered the acute mortality was $2-4 \%$ in those with evidence of RV dysfunction. This dysfunction can initially be assessed by biomarkers such as troponin or N-terminal pro-brain natriuretic peptide (NT-proBNP), right to left ventricular diameter (RV/LV) ratio at end-systole on CTPA and clearly by TTE [4].

The role of precise risk stratification is vital to identify those patients with low-risk PE (figure 2). Therefore, patients with a PESI class I or II, sPESI score of 0 , or meeting no Hestia criteria (with no evidence of RV dysfunction) are considered to have a low-risk PE and are eligible for safe early discharge with careful outpatient management [5]. In the study by BARCO et al. [4] in 2019, this represented 15\% of their cohort.

Patients with haemodynamic instability, i.e. high-risk PE, should be strongly considered for thrombolysis. With reference to intermediate-risk PE, the PEITHO investigators demonstrated no benefit from thrombolysis in the long term; in the immediate setting it does reduce haemodynamic instability but with an increased major bleed risk [6]. There has been much attention given to the use of "reduced-dose" thrombolysis [7, 8]; the 2019 Guidelines, however, still recommend only standard dosing regimens.

In patients with contraindications for thrombolytic therapy, surgical pulmonary embolectomy is still preferred above percutaneous interventional procedures [1]. Prof. Konstantinides was also very clear that extracorporeal membrane oxygenation should only be utilised as a bridge to therapy, i.e. surgical embolectomy or catheter-directed therapy.

The new guidance also states that inferior vena cava filters should only be utilised in two groups of patients: patients with acute $\mathrm{PE}$ and an absolute contraindication to anticoagulation therapy, and patients with well-managed anticoagulation therapy and recurrent PE $[1,9]$.

\section{Pulmonary embolism in pregnancy}

A new section of the guidance, which has been especially well received, is that for PE in relation to pregnancy, as it is the highest cause of maternal death in high-income countries. VTE risk factors in the specific context of pregnancy were underlined, especially the increased risk linked to in vitro fertilisation, particularly in the first trimester [10].

At the 2019 ERS International Congress, the assessment of suspected PE in pregnancy was explained by Guy Meyer (figure 3). By utilising the outcomes of two prospective multicentre studies, a combination of a pregnancy-adapted YEARS algorithm with D-dimer levels substantially increases the probability of safely excluding PE without CTPA $[11,12]$.

Local radiology experience and chest radiography results should aid the decision as to whether to perform CTPA or a scintigraphy scan. The radiation exposure to the fetus is minimal but is high to female breast tissue, particularly with CTPA [13].

Those with a proven PE should be managed in a centre with experience of managing PE in pregnancy. Low-molecular-weight heparin (LMWH) remains the treatment of choice and the dosing regimen should be based on an early pregnancy weight. Also, fondaparinux could be considered if there is an allergy to LMWH. Finally, amniotic fluid embolism should be excluded in cases of severe cardiac or respiratory impairment, especially in the context of disseminated intravascular coagulation.

\section{Cancer and direct oral anticoagulants}

Prof. Meyer underlined the role of direct oral anticoagulants in the treatment and secondary prevention of $\mathrm{PE}$ in patients with cancer $[14,15]$. In patients with non-gastrointestinal cancer and a low risk of bleeding, edoxaban and rivaroxaban can be safely used. For those with active gastrointestinal or genitourinary cancer, LMWH remains the recommended treatment [1]. 


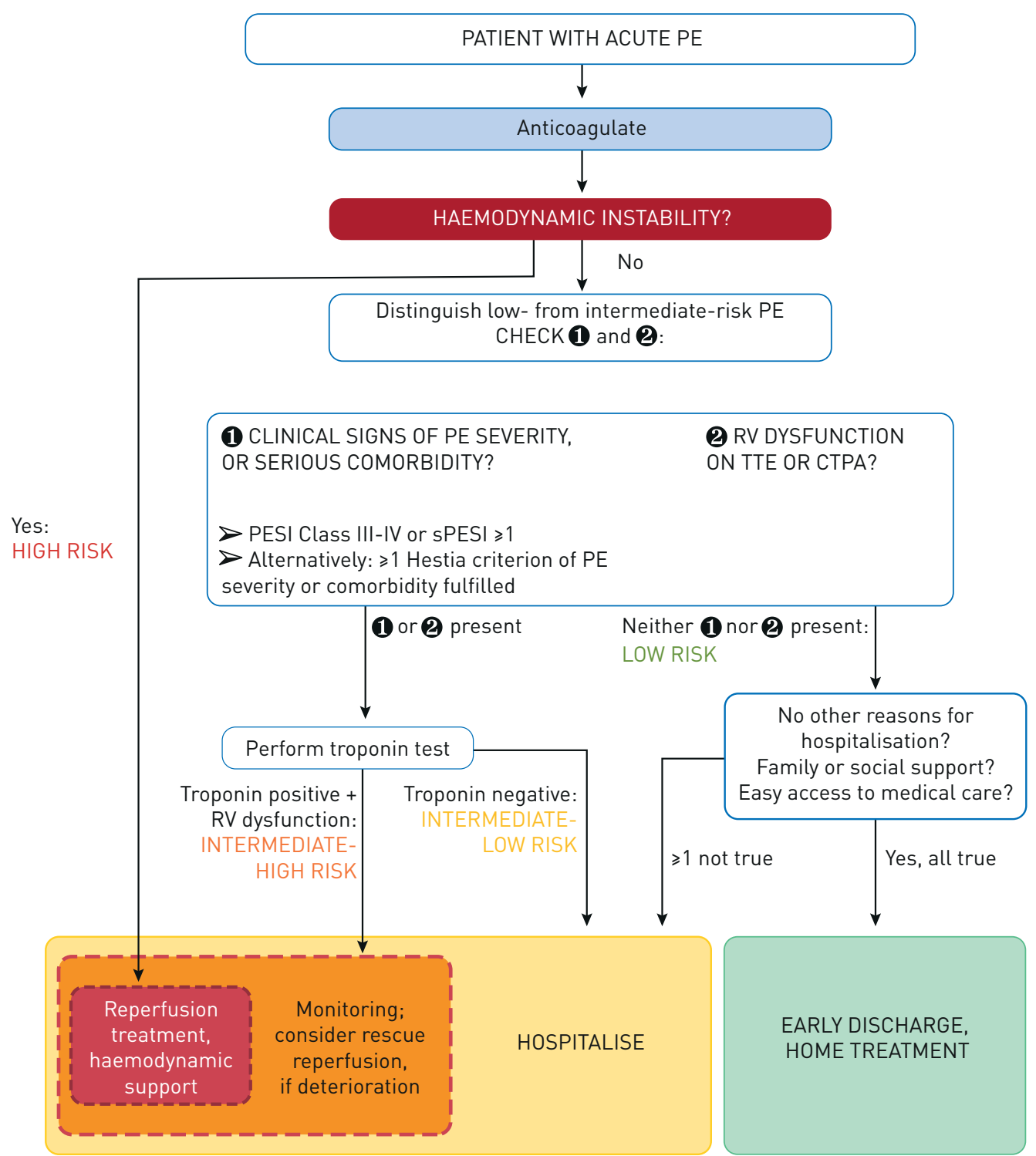

FIGURE 2 Risk-adjusted management strategy for acute pulmonary embolism (PE). RV: right ventricular; TTE: transthoracic echocardiography; CTPA: computed tomography pulmonary angiography; PESI: Pulmonary Embolism Severity Index; SPESI: simplified Pulmonary Embolism Severity Index. Reproduced and modified from [1] with permission.

Extension treatment after 6 months should be strongly considered, especially in those with active cancer. The risk of recurrence of PE in cancer patients was also assessed using a score incorporating factors such as breast or lung cancer, involvement of lymphatic node, female sex and previous VTE [16]. A noteworthy point is that, in patients with cancer, an incidental PE should be managed in the same way as a symptomatic $\mathrm{PE}$, if involving segmental, proximal, multiple subsegmental or a single subsegmental branch in association with a proven deep venous thrombosis.

\section{Pulmonary embolism terminology}

The new Guidelines advise the clinician to avoid terms such as "provoked", "unprovoked" and "idiopathic". The rationale is to prevent potentially erroneous decisions regarding the duration of anticoagulation therapy [1]. The guidance is very clear that the duration of anticoagulation therapy is based on the risk of recurrence of PE and risk of bleeding.

The estimated risk for long-term PE recurrence is divided into three groups: low risk ( $<3 \%$ per year), intermediate risk (3-8\% per year) and high risk ( $>8 \%$ per year). The low-risk group encompasses patients with major transient or reversible factors, e.g. surgery with a general anaesthetic that lasted $>30 \mathrm{~min}$. The intermediate-risk group comprises those with minor transient or reversible factors, such as non-malignant 
SUSPECTED PE DURING PREGNANCY

High pretest probability, or intermediate/low probability and positive D-dimer result

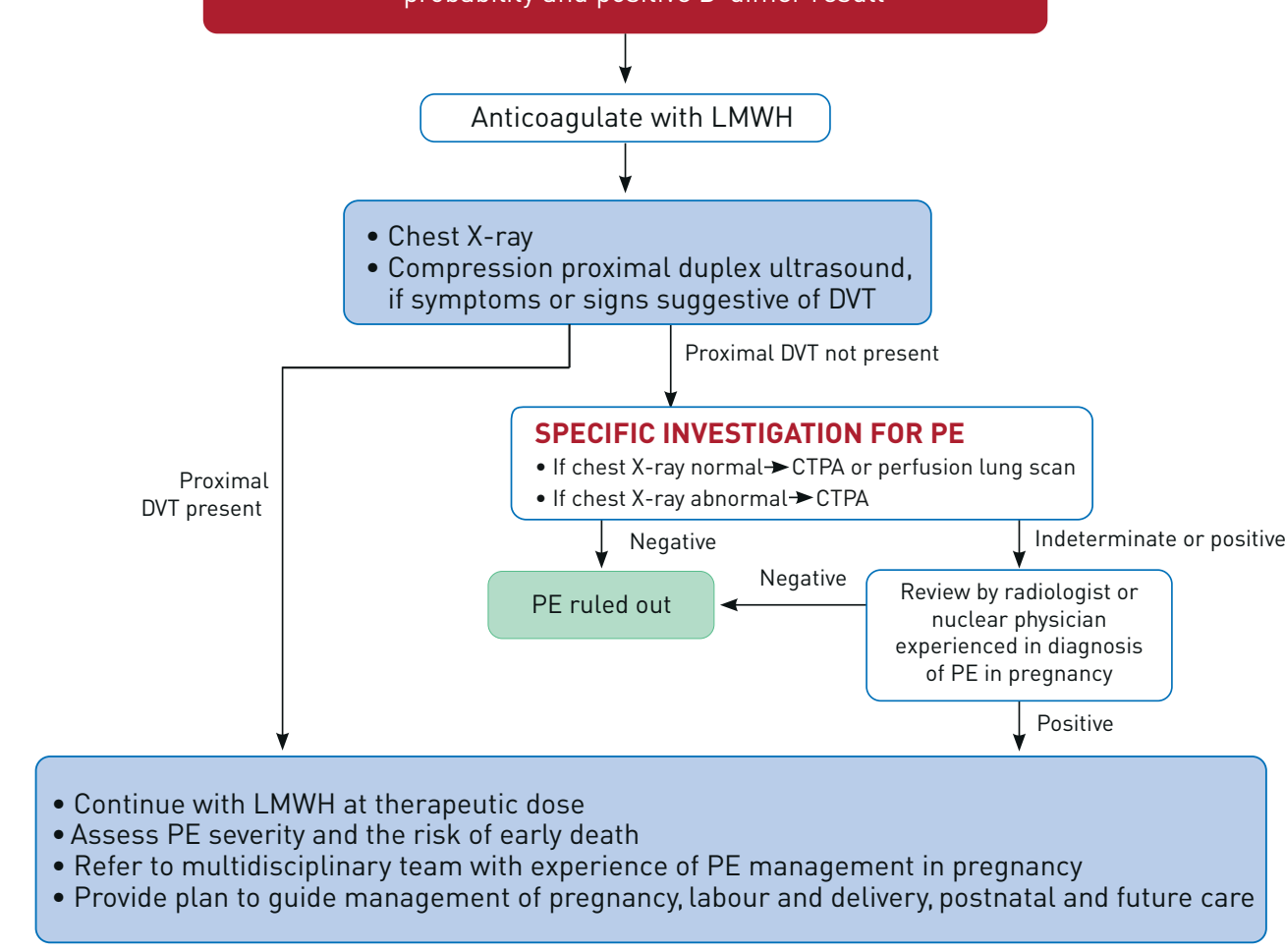

FIGURE 3 Diagnostic work-up and management of suspected pulmonary embolism (PE) during pregnancy and up to 6 weeks post-partum. LMWH: low-molecular-weight heparin; DVT: deep vein thrombosis; CTPA: computed tomography pulmonary angiography. Reproduced and modified from [1] with permission.

persistent factors and cases in whom the index PE event occurred in the absence of any identifiable risk factors, and the high-risk group is composed of patients where risk factors persist, e.g. active cancer [1]

\section{Duration of anticoagulation}

Prof. Meyer echoed the importance of re-assessing patients on anticoagulation therapy at 3 months. The minimum duration of anticoagulation recommended for patients with PE is related to major reversible risk factors. One should consider extension if there is evidence of recurrent VTE not related to a major transient or reversible risk factor, if there is no identifiable risk factor and in those with a persistent risk factor, as well as in patients with a minor transient or reversible risk factor for the index PE event.

When considering extended therapy after 6 months of therapeutic anticoagulation, it is feasible to consider reduced-dose rivaroxaban and apixaban. However, it is important to re-assess the tolerance of the anticoagulant with monitoring of renal and liver function, as well as assessing the bleed risk.

\section{Follow-up}

The Guidelines recommend a follow-up visit at 3-6 months. If there is persistent dyspnoea and/or functional limitation, TTE should be organised, with a cardiopulmonary exercise test also being useful. It is also important to consider risk factors for possible chronic thromboembolic pulmonary hypertension $(\mathrm{CTEPH})$ or chronic thromboembolic disease in both the symptomatic and asymptomatic patient, which are helpfully listed in the new guidance [17-19]. If there is a high probability of pulmonary hypertension $(\mathrm{PH})$ on TTE or evidence of functional limitation, it is recommended to obtain a $V^{\prime} / Q^{\prime}$ scan and to refer the patient to an expert pulmonary vascular disease institute (figure 4).

The recommended CTEPH treatment for proximal disease remains pulmonary endarterectomy. Medical therapy (specifically riociguat) and balloon pulmonary angioplasty are additional therapeutic options for patients with inoperable disease or persistent PH after pulmonary endarterectomy [20, 21]. 


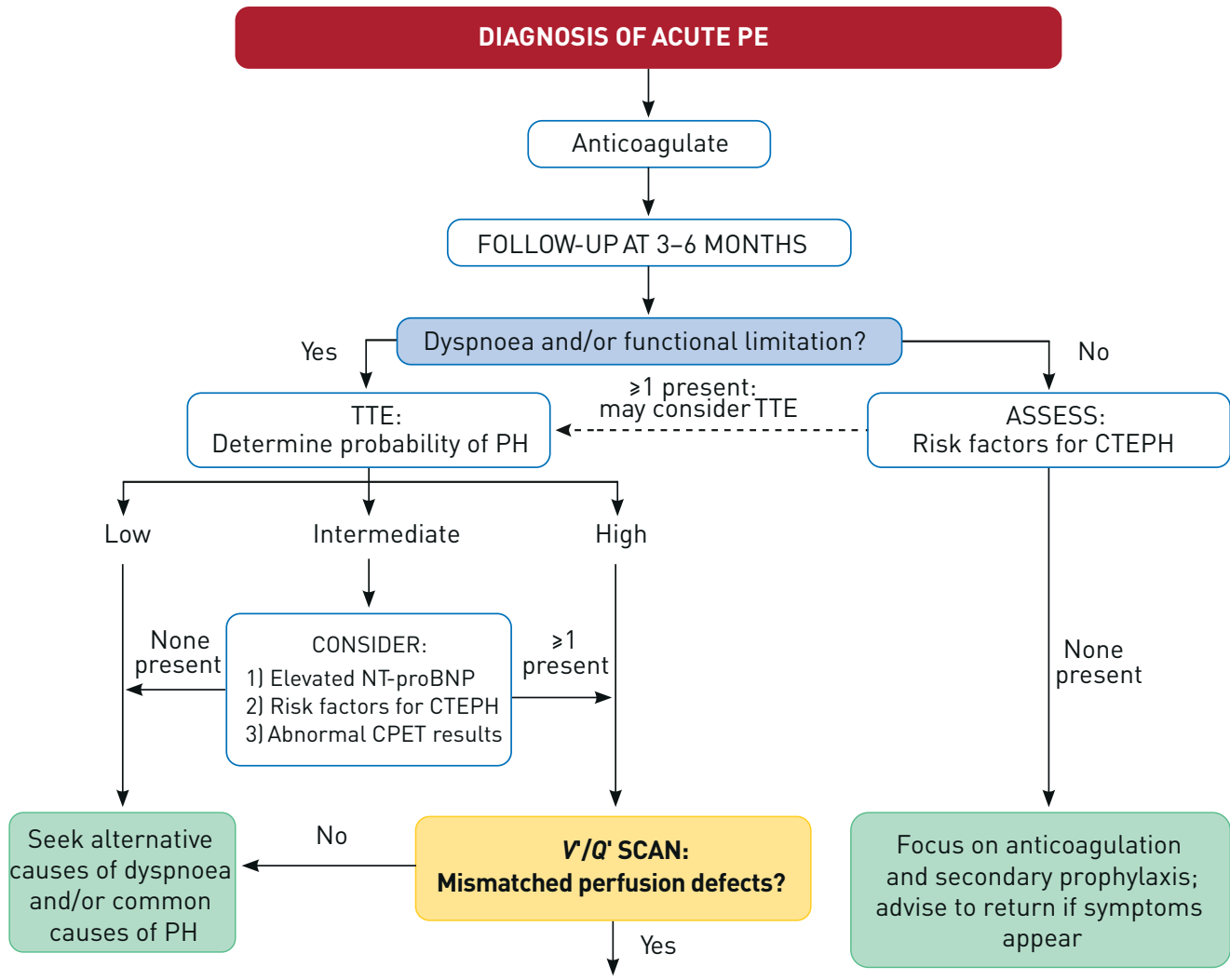

Refer to PH/CTEPH expert

centre for further diagnostic

work-up

FIGURE 4 Follow-up strategy and diagnostic work-up for long-term sequelae of pulmonary embolism (PE). TTE: transthoracic echocardiography; $\mathrm{PH}$ : pulmonary hypertension; $\mathrm{CTEPH}$ : chronic thromboembolic pulmonary hypertension; NT-proBNP: N-terminal pro-brain natriuretic peptide; CPET: cardiopulmonary exercise test; $V^{\prime} / Q^{\prime}$ : ventilation/perfusion. Reproduced and modified from [1] with permission.

\section{Pulmonary hypertension in interstitial lung disease}

$\mathrm{PH}$ frequently affects those with chronic lung disease (CLD). Commonly, there are patients with evidence of mild elevation of pulmonary arterial pressure when measured at echocardiography, but there is also a cohort with indicators of severe $\mathrm{PH}$ that may appear out of proportion to the degree of their CLD. Unsurprisingly, the development of $\mathrm{PH}$ in this cohort of patients is associated with a poorer life expectancy and increased symptom burden, including worse functional capacity, increased oxygen requirements and more frequent acute exacerbations [22].

\section{Prognostic factors}

KAPASI et al. [23] presented a large retrospective study whereby over a 9-year period they identified 5890 patients with idiopathic pulmonary fibrosis (IPF) from their Scientific Registry of Transplant Recipients. These patients were classified into six groups using mean pulmonary arterial pressure (mPAP) and pulmonary vascular resistance (PVR), measured in mmHg and Wood units (WU), respectively: 1) $\mathrm{PH}$ absent (mPAP <21 mmHg); 2) borderline PH, preserved PVR (mPAP 21-24 mmHg, PVR <3 WU); 3) borderline $\mathrm{PH}$, high PVR (PVR $\geqslant 3 \mathrm{WU})$; 4) PH, preserved PVR (mPAP 25-34 mmHg, PVR $<3 \mathrm{WU}$ ); 5) $\mathrm{PH}$, high PVR (PVR $\geqslant 3 \mathrm{WU}$ ); and 6) severe $\mathrm{PH}(\mathrm{mPAP} \geqslant 35 \mathrm{mmHg}$ ). Of their 5890 patients with IPF, $48 \%$ had evidence of $\mathrm{PH}$ and 921 (16\%) had severe PH. Groups 3, 5 and 6 demonstrated that those with a high PVR had an increased risk of death when compared to those with a preserved PVR, suggesting that in IPF patients increased PVR appears to predict poorer survival regardless of the mPAP [23].

This reinforces the study by AwERBACH et al. [24], who showed in interstitial lung disease (ILD)-related PH that PVR $>7$ WU was associated with three-fold higher mortality than PVR $<7$. AwERBACH et al. [24] also demonstrated that ILD-related PH patients, at right heart catheterisation, had lower mPAP and PVR when 
compared to their idiopathic pulmonary arterial hypertension (PAH) cohort; despite these findings, mortality was high in both groups.

Another area of interest is that of pulmonary arterial compliance and its changes during exercise. PANAGIOTIDOU et al. [25] presented the pulmonary arterial compliance of 16 patients with either IPF or ILD related to connective tissue disease. Pulmonary arterial compliance at rest and exercise correlated well with diffusing capacity of the lung for carbon monoxide ( $\left.D_{\mathrm{LCO}}\right)$, 6-min walking distance (6MWD) and New York Heart Association (NYHA) functional class. The decrease in pulmonary arterial compliance may represent a valuable factor in the symptoms and prognosis of ILD-PH and further exploration of this is warranted.

An important noninvasive measure that has previously been reported by BAX et al. [26] is that of RV/LV ratio of $>1$ at CTPA. It is a significantly prognostic indicator of mortality at a multivariate level and appears to be superior to haemodynamic data in this cohort of patients.

\section{Treatment strategies}

The INSTAGE trial disappointingly showed no additional benefit of nintedanib plus sildenafil compared with nintedanib plus placebo in patients with IPF and a $D_{\mathrm{LCO}}$ of $<35 \%$. Its primary end-point was a change in score from baseline in the St George's respiratory questionnaire over a 24 -week period. It did, however, show a numerically lower decline in forced vital capacity in the nintedanib plus sildenafil group [27].

A further sub-analysis of this cohort compared those IPF patients with evidence of right heart dysfunction (RHD) to those without RHD as demonstrated by echocardiography. The investigators found that the IPF group with RHD on nintedanib and sildenafil showed stabilisation of their brain natriuretic peptide (BNP) levels $(p<0.01)$ when compared to the IPF group with no RHD $\left(-119.9 \mathrm{ng} \cdot \mathrm{L}^{-1}, 95 \%\right.$ CI $-171.3--68.5 \mathrm{ng} \cdot \mathrm{L}^{-1}$, and $-3.6 \mathrm{ng} \cdot \mathrm{L}^{-1}, 95 \% \mathrm{CI}-47.2-40.0 \mathrm{ng} \cdot \mathrm{L}^{-1}$, respectively) [28].

Further to this, at the 2019 ERS International Congress, the INSTAGE researchers presented data pertaining to biomarkers relevant to the pathophysiology of IPF, namely biomarkers of inflammation, lung cell damage and extracellular matrix damage. Of the 273 patients treated with nintedanib plus sildenafil, there was an association with statistically significant reductions in collagen 6 degraded by matrix metalloproteinase (MMP)-2/9 and citrullinated vimentin degraded by MMP-2/8, versus nintedanib alone [29].

During the same session, CHANDRAN et al. [30] presented their study findings of the effect of nintedanib on the pulmonary vasculature in a murine model. By utilising Fra2-overexpressing mice (which have a propensity to develop spontaneous ILD and $\mathrm{PH}$ ) and wildtype mice, they were able to ascertain the effects of nintedanib on the pulmonary vasculature by employing wire myography. Nintedanib was shown to induce significant pulmonary artery relaxation, which was endothelium independent and more pronounced in the Fra2 mice. This noteworthy finding certainly warrants further investigation.

According to the preliminary data of the recently completed INCREASE trial of inhaled treprostinil in 326 group $3 \mathrm{PH}$ patients (ClinicalTrials.gov, identifier NCT02630316), this trial has met its primary end-point of an improvement in 6MWD and all its secondary end-points, thus substantiating the careful use of pulmonary vasodilators in appropriate patients with ILD-related PH under the care of expert pulmonary vascular disease centres.

NATHAN et al. [31] have provided a useful algorithm (figure 5) to aid the pulmonologist when deciding which CLD patients should be further assessed at an expert pulmonary vascular disease centre for consideration of PAH therapy on an individualised basis.

\section{Pulmonary arterial hypertension}

\section{Risk stratification}

Risk assessment and prognostication in PAH has been a hot topic in recent years, with many new studies presented at the 2019 ERS International Congress. The updated REVEAL 2.0 risk score was published in 2019, which includes revised cut-off points for some variables compared with the original REVEAL score and now includes the presence of all-cause hospitalisation within the previous 6 months [32]. An external validation of the REVEAL 2.0 was presented using a retrospective cohort of 1011 patients from the Pulmonary Hypertension Society of Australia and New Zealand (PHSANZ) Registry, for which at least seven variables were available [33]. Although lacking information for renal function and NT-proBNP or BNP, REVEAL 2.0 discriminated short-term (12-month) and long-term (60-month) survival in the PHSANZ cohort. There was overlap in survival estimates according to the 8-tier risk strata but excellent discrimination (C-statistic 0.74, 95\% CI 0.72-0.78) using 3-tier risk strata (low, intermediate, high). 


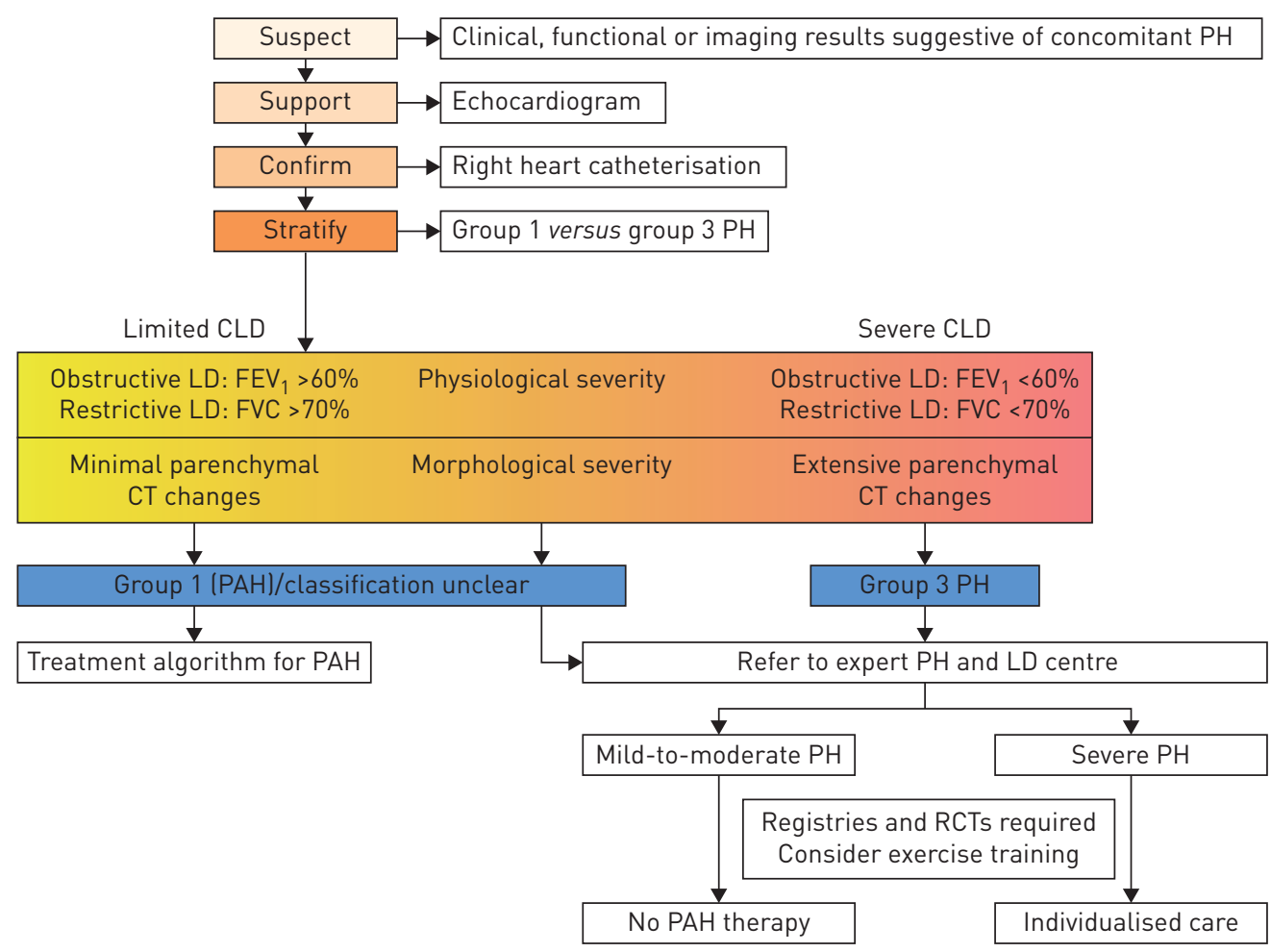

FIGURE 5 Evaluation of pulmonary hypertension (PH) in chronic lung disease (CLD). LD: lung disease; FEV forced expiratory volume in $1 \mathrm{~s}$; FVC: forced vital capacity; CT: computed tomography; PAH: pulmonary arterial hypertension; RCT: randomised controlled trial. Reproduced and modified from [31] with permission.

The EFORT study (ClinicalTrials.gov, identifier NCT01185730) was a prospective, modern cohort of treatment-naïve patients with idiopathic, heritable or drug-induced PAH. The aim of EFORT was to determine transplant-free survival, prognostic factors and treatment goal cut-offs using a dynamic survival prediction model. For 146 patients enrolled between 2012 and 2013, the 1-, 3- and 5-year rates for transplant-free survival were $97 \%, 83 \%$ and $71 \%$, respectively. Increasing age was the only baseline variable independently associated with death or transplantation. Using Cox models with time-dependent variables, optimal treatment goal thresholds identified in this study were NYHA functional class I or II (hazard ratio (HR) $0.19,95 \%$ CI $0.089-0.42$ ), 6MWD $>400 \mathrm{~m}$ (HR 0.16, 95\% CI 0.089-0.42), cardiac index $\geqslant 2.4 \mathrm{~L} \cdot \mathrm{min}^{-1} \cdot \mathrm{m}^{-2} \quad$ (HR $0.16,95 \%$ CI $\left.0.052-0.48\right)$, and either BNP $\leqslant 150 \mathrm{ng} \cdot \mathrm{L}^{-1}$ or NT-proBNP $\leqslant 700 \mathrm{ng} \cdot \mathrm{L}^{-1}$ (HR 0.035 , 95\% CI 0.010-0.12). Achievement of at least two of these goals decreases the instantaneous risk of death or lung transplant, whereas achievement of none or only one of these goals was associated with higher instantaneous risk [34].

There has been limited study on risk stratification in congenital heart disease (CHD)-associated PAH. RAMJUG et al. [35] analysed survival in CHD-PAH patients according to the number of low-risk factors present: NYHA/World Health Organization functional class I/II, incremental shuttle walk test (ISWT) $>420 \mathrm{~m}$, presence of a post-tricuspid defect, and a $D_{\mathrm{LCO}}>60 \%$. The vast majority of patients had none or only one of these low-risk features. In the overall CHD-PAH population $(n=240)$, survival was lowest for patients without any low-risk factors, slightly better for those with one low-risk factor and similar long-term survival in those with two to four low-risk factors [35].

\section{Noninvasive parameters in PAH}

KIELY et al. [36] presented a prospective study that evaluated the reproducibility and sensitivity to change of noninvasive end-points in PAH. They compared changes in biomarkers, 6MWD and the ISWT on repeat tests within $48 \mathrm{~h}$ and at 4-6 months between healthy volunteers and PAH patients. Intraclass correlation was excellent $(>0.74)$ for the $6 \mathrm{MWD}$ and ISWT, as well as for NT-proBNP and almost all magnetic resonance imaging (MRI)-derived volumes and flow measures. For sensitivity to change, the MRI-derived right ventricular ejection fraction (RVEF) had the largest Cohen's D change, whereas 6MWD and NT-proBNP had only fair change, indicating less sensitivity to change. RVEF on MRI may be the most suitable noninvasive end-point for $\mathrm{PAH}$, due to its excellent repeatability and large change in PAH patients undergoing treatment initiation or escalation [36]. 


\section{Use of intravenous prostanoid}

In a retrospective study of $126 \mathrm{PAH}$ patients not at treatment goal with oral therapies, Olsson et al. [37] evaluated the effect of add-on intravenous treprostinil on transplant-free survival and risk strata. Their risk assessment was based on six variables, which were given an integer risk score (1,2 or 3$)$ depending on whether the variable was in the low-, intermediate- or high-risk range. The majority (79\%) were intermediate risk at baseline and $78 \%$ were on double combination therapy. The median treprostinil dose achieved at follow-up was $35 \mathrm{ng} \cdot \mathrm{kg}^{-1} \cdot \mathrm{min}^{-1}$. Long-term transplant-free survival was markedly better for the minority of patients (19\%) who achieved a low-risk status by 6-12 months. However, lack of response within 6-12 months was associated with an approximately 50\% risk of death or transplant in the subsequent 2 years. Haemodynamics did not differ between responders and non-responders. In a multivariable analysis, only $6 \mathrm{MWD}$ and $D_{\mathrm{LCO}}$ predicted a good treatment response. This study suggests that add-on intravenous treprostinil can "rescue" about $20 \%$ of PAH patients but the majority do not obtain treatment targets within 6-12 months and these patients should be considered for lung transplantation, if eligible.

\section{Biomarkers in PAH}

Endostatin is an angiogenic peptide derived from collagen XVIII alpha 1 (Col18a1), an extracellular matrix protein. In recent reports it is correlated with invasive haemodynamics and is shown to be elevated in PAH compared to healthy individuals [38]. Furthermore, a genetic variant in Col18a1 has been associated with differences in serum endostatin levels and outcomes in PAH [39].

At the 2019 ERS International Congress, SIMPSON et al. [40] hypothesised that endostatin is a mechanistic biomarker of disease severity and survival in PAH. They performed whole-genome genotyping and ELISA measurements on 2017 patients with $\mathrm{PAH}$. The investigators found that a higher level of circulating endostatin correlated with higher invasive haemodynamics and lower 6MWD. Additionally, high endostatin levels were independently associated with a poorer prognosis. Based on the sequencing data, the team were also able to demonstrate that serum endostatin levels are influenced by genetic variants in Col18a1, which are consequently associated with phenotypes and outcomes, hence suggesting that endostatin may serve as a biomarker of disease severity in PAH.

Another promising biomarker is asymmetric dimethylarginine (ADMA), which has been shown to be of relevance in different forms of $\mathrm{PH}$ [41-45]. SKORO-SAJER et al. [46] aimed to validate ADMA as a biomarker to monitor disease progression in $\mathrm{PAH}$ and CTEPH patients on targeted PAH therapy or undergoing balloon pulmonary angioplasty. ADMA levels were measured in 47 patients (34 PAH, 13 CTEPH). The patients were stratified into risk groups (low, intermediate, high) according to the ERS/ESC 2015 Guidelines [47]. ADMA did not change significantly between the therapy groups; however, at follow-up in those patients who improved, it correlated with follow-up BNP and risk assessment scores $\left(\mathrm{r}^{2}=0.328\right)$.

Blood count with its broad availability is receiving more attention in the field of biomarker research. Red cell distribution width, for example, has been shown to be prognostic for PAH [48]. The mechanisms that determine the levels of these markers are unclear; however, a study presented at the Congress highlighted the importance of cell-free haemoglobin as a source of oxidative stress [49]. In vitro data on human pulmonary endothelial cells treated with methaemoglobin (metHb) showed that reactive oxygen species (ROS) production and interleukin-6 secretion were increased. This finding implies a strong relationship between metHb-induced oxidated damage and further amplification of endothelial dysfunction through overproduction of cellular ROS.

The prostacyclin pathway is implicated in PAH. It includes prostacyclin, which is associated with vasodilation, and thromboxane, which promotes vasoconstriction. Thromboxane is mainly excreted by platelets; therefore, OLIVEIRA et al. [50] retrospectively analysed 243 patients with PAH at baseline with right heart catheterisation haemodynamics, platelet count and mean platelet volume. When comparing a subset of this cohort to 79 healthy volunteers, they found that PAH individuals had a lower platelet count. Subsequently, platelet count was correlated with an improved survival in the PAH cohort. Conversely, the same Brazilian group demonstrated that $88 \mathrm{CTEPH}$ patients had similar platelet counts to controls, but they showed lower platelet volume. Platelet count or volume was not different between patients who underwent pulmonary endarterectomy and those who were treated conservatively [51].

Iron deficiency is common in those with $\mathrm{PH}$ and conveys a worse prognosis [52-54]. At the Congress, CAMPEAN et al. [55] revealed that there was a high prevalence of iron deficiency in 109 (74\%) CTEPH patients, defined either by ferritin $<100 \mu \mathrm{g} \cdot \mathrm{L}^{-1}$, or by ferritin $100-299 \mu \mathrm{g} \cdot \mathrm{L}^{-1}$ with transferrin saturation $<20 \%$ and raised levels of soluble transferrin receptor $\left(>4.5 \mathrm{nmol} \cdot \mathrm{L}^{-1}\right.$ for females and $>5.0 \mathrm{nmol} \cdot \mathrm{L}^{-1}$ for males). This correlated with lower exercise capacity but not with haemodynamics or survival. 


\section{Noncoding RNA}

Noncoding RNA molecules have gained attention over the last decade as mediators of PH pathogenesis. It has been postulated that they even may become effective targets for future $\mathrm{PH}$ therapies [56, 57]. The noncoding transcriptome can be classified into small noncoding RNAs ( $<200 \mathrm{nt})$, including the microRNAs, and long noncoding RNAs (>200 nt).

Little is known about their role in RV failure. Utilising a rodent model with monocrotaline treatment to induce $\mathrm{PH}$, ConNolly et al. [58] studied the microRNA miR-1, which has been shown to be downregulated in hypertrophying rodent hearts. They were able to clearly demonstrate that transforming growth factor (TGF)- $\beta R 1$ (ALK5) is targeted by miR-1 and miR-1 reduced TGF- $\beta$ activation and its downstream SMAD2/3, proposing that it may regulate cardiac hypertrophy.

A study presented by OMURA et al. [59] utilising RV biopsies from donors with compensated RV hypertrophy (cardiac index $>2.2 \mathrm{~L} \cdot \mathrm{min}^{-1} \cdot \mathrm{m}^{-2}$ ) and decompensated RV hypertrophy (DRVH; PAH patients who died of RV failure), compared to controls, showed that the long noncoding RNA H19 and its encoded microRNA miR-675 were upregulated in DRVH. This was also displayed in a monocrotaline rat model. The team also found that the upregulation of H19/miR-675 was specific to the right ventricle in both rats and humans, as no change was seen in either the left ventricle or lungs of DRVH subjects. They also found in 70 patients with PAH that $\mathrm{H} 19$ was upregulated in plasma and correlated with haemodynamics and prognosis.

Plasma also contains microparticles, which are phospholipid-rich submicron particles that are released from the membranes of endothelial cells, platelets, leukocytes and erythrocytes. MicroRNAs were isolated from microparticles by Ото et al. [60] in control subjects as well as PAH, operable CTEPH and non-operable CTEPH patients. MicroRNA differential expression in microparticles and plasma differed between the four groups. It was noted that miR-133a-3p was found to be dysregulated in both microparticles and plasma, signifying that it may play an important role in $\mathrm{PH}$.

\section{Looking to the future}

In the current pandemic of coronavirus disease 2019, much attention has been given to the topic of the prevention and management of VTE in patients infected with the virus. We are all soon to see if there are any sequelae related to this.

It is hoped that the new ERS/ESC guidance related to the definitions, diagnosis and management of $\mathrm{PH}$ will be realised in 2021. In the meantime, the update articles from the Sixth World Symposium on Pulmonary Hypertension in Nice, France, have provided us with helpful suggestions. As an ERS Assembly, we aspire in the near future, with the advent of the severe Pulmonary Hypertension mAnagement acROSs europe (PHAROS) Clinical Research Collaboration, to be better placed to phenotype our PH patients in order to offer them more personalised and holistic care.

Ultimately, as a community, we have all found innovative ways to connect with one another across Europe and the world. Barriers to progressing with research, both at the bedside and bench side, appear to have been reduced in an attempt to work together as a respiratory community with a common goal. May this ethos continue.

Conflict of interest: S. Ramjug reports nonfinancial support from Janssen, and grants and nonfinancial support from Actelion, during the writing of the manuscript. J. Weatherald reports grants, personal fees and nonfinancial support from Janssen Inc. and Actelion, personal fees and nonfinancial support from Bayer, personal fees from Novartis, and grants from the Alberta Lung Association, the Canadian Vascular Network, the European Respiratory Society and the Canadian Thoracic Society, outside the submitted work. S. Sahay reports personal fees and nonfinancial support from Bayer Pharmaceuticals, United Therapeutics, Actelion Pharmaceuticals, and Liquidia. J. Khoury has nothing to disclose. V. Foris has nothing to disclose. N. Chandran has nothing to disclose. A. Bokan has nothing to disclose. L. Godinas has nothing to disclose. M. Delcroix reports grants and personal fees from Actelion, and personal fees from Bayer, GSK, MSD, Reata, Bellarophon and Eli Lilly, outside the submitted work.

\section{References}

1 Konstantinides SV, Meyer G, Becattini C, et al. 2019 ESC Guidelines for the diagnosis and management of acute pulmonary embolism developed in collaboration with the European Respiratory Society (ERS). Eur Respir J 2019; 54: 1901647.

2 Righini M, Van Es J, Den Exter PL, et al. Age-adjusted D-dimer cutoff levels to rule out pulmonary embolism: the ADJUST-PE study. JAMA 2014; 311: 1117-1124.

3 van der Hulle T, Cheung WY, Kooij S, et al. Simplified diagnostic management of suspected pulmonary embolism (the YEARS study): a prospective, multicentre, cohort study. Lancet 2017; 390: 289-297.

4 Barco S, Mahmoudpour SH, Planquette B, et al. Prognostic value of right ventricular dysfunction or elevated cardiac biomarkers in patients with low-risk pulmonary embolism: a systematic review and meta-analysis. Eur Heart J 2019; 40: 902-910.

5 Howard LSGE, Barden S, Condliffe R, et al. British Thoracic Society Guideline for the initial outpatient management of pulmonary embolism (PE). Thorax 2018; 73: Suppl. 2, ii1-ii29. 
6 Meyer G, Vicaut E, Danays T, et al. Fibrinolysis for patients with intermediate-risk pulmonary embolism. $N$ Engl J Med 2014; 370: 1402-1411.

7 Layman SN, Guidry TJ, Gillion AR. Low-dose alteplase for the treatment of submassive pulmonary embolism: a case series. J Pharm Pract 2019; in press [https://doi.org/10.1177/0897190019855164].

8 Kiser TH, Burnham EL, Clark B, et al. Half-dose versus full-dose alteplase for treatment of pulmonary embolism. Crit Care Med 2018; 46: 1617-1625.

9 Bikdeli B, Chatterjee S, Desai NR, et al. Inferior vena cava filters to prevent pulmonary embolism: systematic review and meta-analysis. J Am Coll Cardiol 2017; 70: 1587-1597.

10 Henriksson $\mathrm{P}$, Westerlund $\mathrm{E}$, Wallén $\mathrm{H}$, et al. Incidence of pulmonary and venous thromboembolism in pregnancies after in vitro fertilisation: cross sectional study. BMJ 2013; 346: e8632.

11 Righini M, Robert-Ebadi H, Elias A, et al. Diagnosis of pulmonary embolism during pregnancy a multicenter prospective management outcome study. Ann Intern Med 2018; 169: 766-773.

12 van der Pol LM, Tromeur C, Bistervels IM, et al. Pregnancy-adapted YEARS algorithm for diagnosis of suspected pulmonary embolism. N Engl J Med 2019; 380: 1139-1149.

13 Perisinakis K, Seimenis I, Tzedakis A, et al. Perfusion scintigraphy versus 256-slice CT angiography in pregnant patients suspected of pulmonary embolism: comparison of radiation risks. J Nucl Med 2014; 55: $1273-1280$.

14 Raskob GE, van Es N, Verhamme $\mathrm{P}$, et al. Edoxaban for the treatment of cancer-associated venous thromboembolism. N Engl J Med 2018; 378: 615-624.

15 Young AM, Marshall A, Thirlwall J, et al. Comparison of an oral factor Xa inhibitor with low molecular weight heparin in patients with cancer with venous thromboembolism: results of a randomized trial (SELECT-D). J Clin Oncol 2018; 36: 2017-2023.

16 Louzada ML, Carrier M, Lazo-Langner A, et al. Development of a clinical prediction rule for risk stratification of recurrent venous thromboembolism in patients with cancer-associated venous thromboembolism. Circulation 2012; 126: 448-454

17 Pepke-Zaba J, Delcroix M, Lang I, et al. Chronic thromboembolic pulmonary hypertension (CTEPH): results from an international prospective registry. Circulation 2011; 124: 1973-1981.

18 Lang IM, Simonneau G, Pepke-Zaba JW, et al. Factors associated with diagnosis and operability of chronic thromboembolic pulmonary hypertension. A case-control study. Thromb Haemost 2013; 110: 83-91.

19 Bonderman D, Wilkens H, Wakounig S, et al. Risk factors for chronic thromboembolic pulmonary hypertension. Eur Respir J 2009; 33: 325-331.

20 Bernardo RJ, Bokan A, Ramjug S. Updates in pulmonary hypertension and other pulmonary vascular diseases. Breathe 2019; 15: 241-243.

21 Wilkens H, Lang I, Behr J, et al. Chronic thromboembolic pulmonary hypertension (CTEPH): updated recommendations of the Cologne Consensus Conference 2011. Int J Cardiol 2011; 154: Suppl. 1, S54-S60.

22 King CS, Nathan SD. Pulmonary hypertension due to interstitial lung disease. Curr Opin Pulm Med 2019; 25 $459-467$.

23 Kapasi A, Halloran K, Hirji A, et al. Elevated pulmonary vascular resistance is associated with increased risk of death in IPF. Eur Respir J 2019; 54: Suppl. 63, PA1428.

24 Awerbach JD, Stackhouse KA, Lee J, et al. Outcomes of lung disease-associated pulmonary hypertension and impact of elevated pulmonary vascular resistance. Respir Med 2019; 150: 126-130.

25 Panagiotidou E, Stanopoulos I, Papakosta D, et al. Rest and exercise pulmonary arterial compliance in patients with pulmonary fibrosis. Eur Respir J 2019; 54: Suppl. 63, PA1429.

26 Bax S, Jacob J, Ahmed R, et al. Right ventricular to left ventricular ratio at CT pulmonary angiogram predicts mortality in interstitial lung disease. Chest 2020; 157: 89-98.

27 Kolb M, Raghu G, Wells AU, et al. Nintedanib plus sildenafil in patients with idiopathic pulmonary fibrosis. N Engl J Med 2018; 379: 1722-1731.

28 Behr J, Kolb M, Song JW, et al. Nintedanib and sildenafil in patients with idiopathic pulmonary fibrosis and right heart dysfunction. A prespecified subgroup analysis of a double-blind randomized clinical trial (INSTAGE). Am J Respir Crit Care Med 2019; 200: 1505-1512.

29 White E, Crestani B, Günther A, et al. Changes in biomarkers in patients with idiopathic pulmonary fibrosis (IPF) treated with nintedanib and sildenafil. Eur Respir J 2019; 54: Suppl. 63, OA1923.

30 Chandran N, Sharma N, Wollin L, et al. Nintedanib has acute pulmonary vasodilatory effects in transgenic Fra2 mice with spontaneous progressive pulmonary hypertension and lung fibrosis. Eur Respir J 2019; 54: Suppl. 63, PA5382.

31 Nathan SD, Barbera JA, Gaine SP, et al. Pulmonary hypertension in chronic lung disease and hypoxia. Eur Respir J 2019; 53: 1801914.

32 Benza RL, Gomberg-Maitland M, Elliott CG, et al. Predicting survival in patients with pulmonary arterial hypertension: the REVEAL risk score calculator 2.0 and comparison with ESC/ERS-based risk assessment strategies. Chest 2019; 156: 323-337.

33 Anderson JJ, Lau EM, Lavender M, et al. Retrospective validation of the REVEAL 2.0 risk score with the Australian and New Zealand Pulmonary Hypertension Registry cohort. Chest 2020; 157: 162-172.

34 Sitbon $\mathrm{O}$, Clerson $\mathrm{P}$, Cottin V, et al. A dynamic prognostic model to predict survival and determine treatment goals in pulmonary arterial hypertension (PAH): the EFORT study. Eur Respir J 2019; 54: Suppl. 63, OA497.

35 Ramjug S, Billings C, Bowater S, et al. Risk stratification of pulmonary arterial hypertension (PAH) associated with adult congenital heart disease (ACHD). Eur Respir J 2019; 54: Suppl. 63, OA498.

36 Kiely D, Swift A, Cogliano M, et al. A prospective study comparing the repeatability and sensitivity to change of non-invasive endpoints in pulmonary arterial hypertension: the RESPIRE study. Eur Respir J 2019; 54: Suppl. 63 , OA496.

37 Olsson KM, Richter MJ, Kamp JC, et al. Intravenous treprostinil as an add-on therapy in patients with pulmonary arterial hypertension. J Heart Lung Transplant 2019; 38: 748-756.

38 Hoffmann J, Marsh LM, Pieper M, et al. Compartment-specific expression of collagens and their processing enzymes in intrapulmonary arteries of IPAH patients. Am J Physiol Lung Cell Mol Physiol 2015; 308: L1002-L1013. 
Damico R, Kolb TM, Valera L, et al. Serum endostatin is a genetically determined predictor of survival in pulmonary arterial hypertension. Am J Respir Crit Care Med 2015; 191: 208-218.

Simpson C, Damico RL, Hassoun PM, et al. Serum endostatin as a genetically-influenced biomarker in PAH. Eur Respir J 2019; 54: Suppl. 63, OA500.

Liu J, Fu Q, Jiang L, et al. Clinical value of asymmetrical dimethylarginine detection in patients with connective tissue disease-associated pulmonary arterial hypertension. Cardiol Res Pract 2019; 2019: 3741909.

Siques P, Brito J, Schwedhelm E, et al. Asymmetric dimethylarginine at sea level is a predictive marker of hypoxic pulmonary arterial hypertension at high altitude. Front Physiol 2019; 10: 651.

Fang Z, Huang Y, Tang L, et al. Asymmetric dimethyl-L-arginine is a biomarker for disease stage and follow-up of pulmonary hypertension associated with congenital heart disease. Pediatr Cardiol 2015; 36: 1062-1069.

Gorenflo M, Zheng C, Werle E, et al. Plasma levels of asymmetrical dimethyl-L-arginine in patients with congenital heart disease and pulmonary hypertension. J Cardiovasc Pharmacol 2001; 37: 489-492.

Skoro-Sajer N, Mittermayer F, Panzenboeck A, et al. Asymmetric dimethylarginine is increased in chronic thromboembolic pulmonary hypertension. Am J Respir Crit Care Med 2007; 176: 1154-1160.

Skoro-Sajer N, Probst V, Sadushi-Kolici R, et al. Asymmetric dimethylarginine (ADMA) to monitor treatments of pulmonary hypertension. Eur Respir J 2019; 54: Suppl. 63, OA501.

Galiè N, Humbert M, Vachiery JL, et al. 2015 ESC/ERS Guidelines for the diagnosis and treatment of pulmonary hypertension. Eur Respir J 2015; 46: 903-975.

Rhodes CJ, Wharton J, Howard LS, et al. Red cell distribution width outperforms other potential circulating biomarkers in predicting survival in idiopathic pulmonary arterial hypertension. Heart 2011; 97: 1054-1060.

Toe Q, Mohd Ghazaly M, Issit TJ, et al. Cell free haemoglobin and pulmonary artery endothelial cell dysfunction. Eur Respir J 2019; 54: Suppl. 63, PA5042.

0 Oliveira T, Calderaro D, Piloto B, et al. Platelets and pulmonary arterial hypertension (PAH). Eur Respir J 2019; 54: Suppl. 63, PA4756.

1 Oliveira T, Kato-Morinaga L, Assad A, et al. Platelets and chronic thromboembolic pulmonary hypertension. Eur Respir J 2019; 54: Suppl. 63, PA1444.

2 Rhodes CJ, Howard LS, Busbridge M, et al. Iron deficiency and raised hepcidin in idiopathic pulmonary arterial hypertension: clinical prevalence, outcomes, and mechanistic insights. J Am Coll Cardiol 2011; 58: 300-309.

Lakhal-Littleton S, Crosby A, Frise MC, et al. Intracellular iron deficiency in pulmonary arterial smooth muscle cells induces pulmonary arterial hypertension in mice. Proc Natl Acad Sci USA 2019; 116: 13122-13130.

Soon E, Treacy CM, Toshner MR, et al. Unexplained iron deficiency in idiopathic and heritable pulmonary arterial hypertension. Thorax 2011; 66: 326-332.

Campean IA, Sadushi-Kolici R, Beckmann HM, et al. Iron deficiency in chronic thromboembolic pulmonary hypertension. Eur Respir J 2019; 54: Suppl. 63, PA1443.

6 Kung JTY, Colognori D, Lee JT. Long noncoding RNAs: past, present, and future. Genetics 2013; 193: 651-669.

Negi V, Chan SY. Discerning functional hierarchies of microRNAs in pulmonary hypertension. JCI Insight 2017; 2: e91327.

8 Connolly M, Wort S, Garfield B, et al. MiR-1-5p targets TGF- $\beta$ R1 and is suppressed in the hypertrophying hearts of rats with pulmonary arterial hypertension. Eur Respir J 2019; 54: Suppl. 63, PA5045.

9 Omura J, Habbout K, Martineau S, et al. Long non-coding RNA H19 in right ventricular failure associated with pulmonary arterial hypertension. Eur Respir J 2019; 54: Suppl. 63, PA5040.

Oto J, Tura-Ceide O, Sánchez-López V, et al. Differential expression of miRNAs present in plasma and contained within circulating microparticles in precapillary pulmonary hypertension. Eur Respir J 2019; 54: Suppl. 63, PA5050. 